Journal of Case Reports 2017;7(4):332-334

\title{
Heart in Brain
}

\author{
TCR Ramakrishnan, C Kanagaraju, S Akhtar, A Patel
}

Department of Neurology, K.G. Hospital and Postgraduate Institute and Karpagam Faculty of Medical Sciences and Research, Coimbatore, Tamil Nadu, India.

\section{Corresponding Author:}

Dr. Saleem Akhtar

Email: salim.ak06@gmail.com

This is an Open Access article distributed under the terms of the Creative Commons Attribution License (creativecommons.org/ licenses/by/3.0).

Received : June 8, 2017

Accepted : August 30, 2017

Published : October 10,2017

\begin{abstract}
Background: Bilateral medial medullary syndrome is a rare type of stroke which results due to occlusion of the anterior spinal artery or vertebral artery or its branches. Clinical diagnosis without neuroimaging is very difficult, the characteristic brain MRI finding of "heart appearance" at DWI has been described in bilateral medial medullary infarct. Case Report: We hereby report 52 year old with acute onset quadriparesis. MRI scan of brain, diffusion weighted (DW) imaging revealed heart shaped hyper-intensity areas in the bilateral medial medulla. Conclusion: MRI with diffusion-weighted imaging helps in timely diagnosis of bilateral MMI in the acute setting and consequently decreasing the possibility of long-term disability.
\end{abstract}

Keywords: Brain, Brain Stem Infractions, Medial Medullary Syndrome, Neuroimaging, Quadriplegia.

\section{Introduction}

Medial medullary infarction is an uncommon stroke and represents less than $1 \%$ of posterior circulation strokes associated generally with a poor prognosis. Bilateral medial medullary infarction (MMI) is even a rarer stroke syndrome and only a handful of cases have been described. The classical signs of MMI consist of contralateral hemiparesis, lemeniscal sensory loss, ipsilateral lingual palsy and contralateral pharyngeal palsy. Patients with bilateral MMI syndrome often present with tetraparesis, bilateral loss of deep sensation, dysphagia and speech difficulties. Diagnosis of bilateral MMI has become easily possible with the advent of Diffusion-Weighted magnetic resonance imaging (DW-MRI). We report the case of a 52 year old patient who was diagnosed with bilateral MMI based on clinical and MRI features. We are presenting this case due to its rarity, only 12 anatomically proven cases are reported in literature so far.

\section{Case Report}

52 year old male patient, known diabetic and hypertensive, presented with history of acute onset of weakness of all four limbs. This weakness worsened over the ensuing 24 hours with slurring of speech. There was no history of difficulty in chewing, headache, vomiting, seizure or loss of consciousness. There was no history of any febrile episode in recent time or diarrheal illness or trauma in recent time.

On neurologic examination, higher mental functions were normal. Cranial nerve examination revealed right upper motor neuron VII nerve palsy with bilateral lower motor neuron $12^{\text {th }}$ nerve palsy (right $>$ left). Remaining central nervous examination was essentially normal. Further examination revealed mild hypertonia with quadriparesis with power of $1 / 5$ in all limbs, brisk deep tendon reflexes and bilateral extensor plantar responses. No sensory impairment was noted. Cerebellum and 
gait could not be examined. History and physical examination localized the lesion to brainstem at medullary level. Hematological investigations did not show any significant abnormality. MRI scan of brain, diffusion weighted (DW) imaging revealed heart shaped hyper-intensity areas in the bilateral medial medulla as well as T2-weighted imaging suggestive of acute medial medullary infarct [Fig.1A,B].

Patient was treated with loading dose of aspirin and clopidogrel and statin followed by 75 $\mathrm{mg}$ of aspirin and $75 \mathrm{mg}$ of clopidogrel and $40 \mathrm{mg}$ of atorvastatin along with deep vein thrombosis prophylaxis and supportive measures.

\section{Discussion}

Medial medullary syndrome is a rare stroke subtype characterized by ipsilateral hypoglossal nerve palsy, contralateral hemiparesis sparing face and impairment of contralateral deep sensation. It results from infarction of para-median region of medulla oblongata due to occlusion of vertebral/ anterior spinal artery or their small branches. Medial medullary infarction is a rare occurrence and represents $<1 \%$ of posterior circulation strokes [1]. Bilateral MMI is even rarer stroke subtype and only 12 anatomically proven cases have been reported in English literature till date [2]. Subsequently, with advances in imaging technology with widespread use of MRI in diagnosis of acute ischemic strokes worldwide, additional 38 cases have been reported in the last 20 years. Katoh and Kawamoto classified bilateral MMI into type I, with an infarction area from medullary pyramid to pontine medial longitudinal fasciculus and type II with infarction confined to bilateral medullary pyramids [3]. It has generally a poor prognosis [4-6]. The vascular events likely to be associated with bilateral MMI are occlusion of vertebral artery or anterior spinal artery and its intrinsic penetrating branches. The infarcted area usually includes the pyramidal tracts, medial lemniscus, medial longitudinal fasciculus, hypoglossal nucleus or
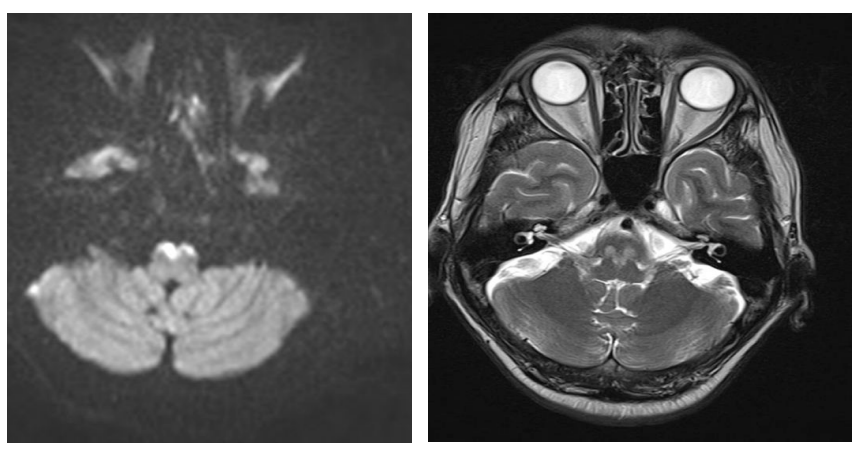

Fig.1(A): Diffusion weighted image. (B): T2 weighted image.

hypoglossal nerve fibres and medullary reticular formation bilaterally [4]. The medulla oblongata has a vast and unique vascular network and its arterial supply arises from the anterior and posterior spinal arteries in addition to the perforating arteries and the long circumferential artery that arise from the basilar or vertebral arteries $[7,8]$. Infarcts involving the medulla oblongata are categorized on the basis of its vascular supply into four territories: anteriormedial territory, anterior-lateral territory, lateral territory, and posterior territory $[8,9]$.

The 'heart-sign' is believed to appear when the former two regions are involved [7]. Misdiagnosis/delay in diagnosis in this syndrome is common as patient presenting with areflexic quadriplegia sparing face may be misdiagnosed as Guillain-Barre syndrome, myasthenia gravis, brainstem encephalitis, inflammatory myopathy, periodic paralysis and para-neoplastic syndrome as other possible differential diagnosis. Bilateral MMI should be suspected in patients presenting with acute onset quadriparesis, tongue weakness and facial sparing. Limb weakness, respiratory failure, aspiration pneumonia, uro-sepsis and pulmonary thromboembolism are important cause of mortality and seen in significant number of cases [2]. Computed tomography scan of brain is not a sensitive tool for posterior fossa, especially for medullary infarcts as in our case, CT scan of brain was normal even after three days of onset of symptoms. DWI sequence of MRI shows the characteristic heart appearance sign due to 
infarction of antero-medial and antero-lateral territory of medulla. Acute to sub-acute MMI can be differentiated accurately only by MRI. In acute MMI, there will be diffusion restriction with ADC reversal and no abnormality on T2/FLAIR sequences. Sub-acute MMI shows T2 shine through phenomena, no ADC reversal with hyper-intense signal changes on T2/FLAIR [10]. Intravenous/ intra-arterial thrombolysis using recombinant tissue plasminogen activator ( $r$-TPA) may be beneficial in cases with bilateral MMI. Pfefferkorn et al. have demonstrated that a combination of intravenous thrombolysis with consecutive in endovascular mechanical thrombectomy may be an option in this difficult clinical situation [11].

In summary bilateral MMI is a rare stroke presenting with rapid onset quadriplegia with sensory loss and bulbar weakness which can also be seen in patients with no known co-morbidities, these clinical features should raise suspicion of bilateral MMI.

\section{Conclusion}

Advances in imaging technology like MRI with diffusion-weighted imaging helps in timely diagnosis of bilateral MMI in the acute setting and consequently decreasing the possibility of longterm disability.

Contributors: TCRR, CK: literature search, case management; SA, AP: manuscript editing, literature search. SA will act as guarantor. All authors approved the final version of the manuscript.

Funding: None; Competing interests: None stated.

\section{References}

1. Bassetti C, Bogousslavsky J, Mattle H, Bernasconi A. Medial medullary stroke: report of seven patients and review of the literature. Neurology. 1997;2013:882-890.

2. Toyoda K, Hasegawa Y, Yonehara T, Oita J, Yamaguchi T. Bilateral medial medullary infarction with oculomotor disorders. Stroke. 1992;23:1657-1659.

3. Katoh M, Kawamoto T. Bilateral medial medullary infarction. J Clin Neurosci. 2000;7:543-545.

4. Kumral E, Afsar N, KIrbas D, BalkIr K, T. ÖzdemirkIran T. Spectrum of medial medullary infarction: clinical and magnetic resonance imaging findings. Journal of Neurology. 2002;249;85-93.

5. Kleinert G, Fazekas F, Kleinert R, Schmidt R, Payer $\mathrm{F}$, Offenbacher $\mathrm{H}$, et al. Bilateral medial medullary infarction: magnetic resonance imaging and correlative histopathologic findings. European Neurology. 1993;33:74-76.

6. Jagiella WM, Sung JH. Bilateral infarction of the medullary pyramids in humans. Neurology. 1989;39:2124.

7. Tokuoka K, Yuasa N, Ishikawa T, Takahashi M, Mandokoro H, Kitagawa Y, et al. A case of bilateral medial medullary infarction presenting with "heart appearance" sign. Tokai J Exp Clin Med. 2007;32:99102.

8. Tatu L, Moulin T, Bogousslavsky J, Duvernoy H. Arterial territories of human brain: brainstem and cerebellum. Neurology. 1996;47:1125-1135.

9. Duvernoy HM, Vannson JL. Human brain stem vessels: including the pinal gland and information on brain stem infarction. $2^{\text {nd }}$ ed. New York, Springer-Verlag 1999.

10. Pongmoragot J, Parthasarathy S, Selchen D, Saposnik G. Bilateral medial medullary syndrome. A systematic review. Journal of Stroke and Cerebrovascular Diseases. 2013;22:775-780.

11. Pfefferkorn T, Mayer TE, Opherk C, Peters N, Straube A, Pfister HW, et al. Staged escalation therapy in acute basilar artery occlusion: intravenous thrombolysis and on-demand consecutive endovascular mechanical thrombectomy: preliminary experience in 16 patients. Stroke. 2008;39:1496-1500. 